\title{
TÜRKISCHE PFERDEWÖRTER BEI EINEM SANSKRIT-LEXIKOGRAPHEN?
}

\author{
von \\ HERMANN BERGER \\ Heidelberg
}

In der Festgabe für Herman Lommel (Paideuma, Bd. VII, Heft 4/6, Juli 1960, p. 274-76) hat Manfred Mayrhofer unter dem Titel "Mongolische Pferdewörter bei einem Sanskrit-Lexikographen?" aus Hemacandras Abhidhänacintämani (12. Jh.) acht Wörter für Pferde mit bestimmten Farben zusammengestellt, die alle auf -äha enden und auffallende etymologische Entsprechungen im Mongolischen haben. Ich lasse die Gleichungen der Bequemlichkeit halber noch einmal hier in der von Mayrhofer gegebenen Form folgen:

1. kulāha- (H. 1241) "hellbraunes Pferd mit schwarzen Knien" (manākpītah krșnạn syäd yadi jānuni);

$\sim$ (schrift)mongol. qula, mittelmongol. qula, khalkha $\chi u l a$, burjät. $\chi$ ula "braun (als Pferdebezeichnung)", Pp ${ }^{1}$.; Pch. ${ }^{1}$ verweist noch besonders auf qulan "gelblich braunes Wildpferd, Przewalski-Pferd".

2. kokãha-(H. 1237) "Schimmel” (H.: site tu karka-kokähau [2 Wörter]; $\sim$ mongol. köke, kalmück. $k \ddot{b} k^{\ddot{o}}$, mittelmong. köke, khalkha $\chi \ddot{o} \chi \ddot{o}$ u.a. "grau, himmelfarben, bläulich, blau, grün"; Pp., Pch.

3. khoñgäha- (H. 1237) "Braunschecke, weiß-und-braunes Pferd" (śvetapingale);

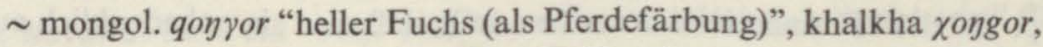

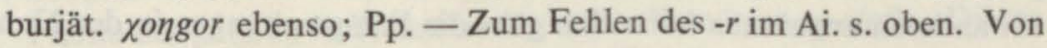
diesem wohl nicht zu trennen ist:

4. khuñgäha- (H. 1238), dem Hemacandra freilich die Bedeutung "Rappe, dunkles Pferd" zuschreibt (krșnavarne tu khuñgāhah), die zu den mongolischen Bedeutungen nicht exakt stimmt.

5. serāha- (H. 1238) "milchweißes Pferd" (pĩyūșavarnee); mongol. šira "gelb", khalkha, burjät., kalmück. šara dss.; Pp., Pch. 6. halāha- (H. 1243) "Schecke" (halāhaś citrito hayah);

1 Nikolaus N. Poppe und Pavel Poucha, Mayrhofers mongolistische Berater. 
$\sim$ mittelmong. halaq (schriftmong. ala $\gamma$, khalkha alag u.a) "scheckig", Pp. - Zum Fehlen des $-q$ im Ai. s. oben.

Nicht ganz so glatt sind die beiden restlichen Vergleiche:

7. ukanāha- (H. 1241) "rötlichbraunes, kastanienbraunes Pferd" (pītaraktacchāyah);

mongol. u $\chi \bar{a}$ "hellkastanienfarbig (als Pferdefärbung)" paßt der Bedeutung nach ausgezeichnet (kommt im Khalkha und Burjätischen vor; Pp.) -nä- im Hemacandra-Wort fehlt jedoch dem Mongolischen. 8. vollāha- (H. 1239) "kastanienbraunes Pferd, bei dem Mähne und Schweif hell sind" (vollähas tv ayam eva syāt pāndukeśaravāladhih);

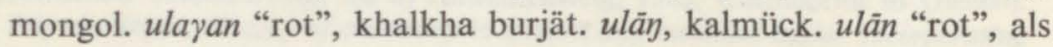
Pferdefärbung "ein hellgraues Pferd mit bräunlichen Tüpfeln" (Pp.), ist nach Form und Bedeutung nicht ganz passend. Pch. geht von *boll ${ }^{\circ}$ im Sanskrit aus, mit dem bekannten Wechsel $b / v$, und verweist auf kalmück. borḷā "grau" (als Farbe von Menschen, Pferden und Vögeln).

Beim Durchblättern von Ilse Laude-Cirtautes' Arbeit Der Gebrauch der Farbbezeichnungen in den Türkdialekten ${ }^{2}$ fand ich für fast alle der genannten Nomina auch türkische Entsprechungen. Bei den regen Lehnbeziehungen, die zu allen Zeiten zwischen den türkischen und mongolischen Sprachen bestanden, könnte daraus keineswegs zwingend auf das Türkische als Quelle der Sanskritwörter geschlossen werden. Aber das Türkische bietet nicht nur sichere Entsprechungen von zwei Wörtern, die Mayrhofer nicht aus dem Mongolischen deuten konnte (triyūha und surūhaka), sondern erlaubt auch eine Erklärung des Elementes -äha, das Mayrhofer wenig überzeugend als eine Sanskritisierung von neuind. $-\bar{a}$ gedeutet hat (p. 275): Varianten mit suffixaler Erweiterung durch einen velaren Spiranten sind bei drei Wörtern unserer Liste belegbar $(1,5,6)^{3}$ und im Übrigen bei Farbadjektiven in den Turksprachen so häufig (vgl. p. 120 des "Formantienindexes" bei LC), daß man sie unbedenklich auch bei anderen annehmen kann. Ich gebe die Entsprechungen in der Numerierung Mayrhofers:

1. kulāha: türk. qula "braun, grau" ist in der Bedeutung "falb mit schwarzer Mähne und schwarzem Schwanze", "braunes Pferd mit schwarzem Schwanze und ebensolcher Mähne", "Pferd mit gelben Ringen an den Füßen und schwarzen Streifen am Rücken" belegt und scheint

2 Wiesbaden 1961; ab jetzt als LC abgekürzt. Mit "türkisch" is in der Folge nie das Türkeitürkische, sondern die Gesamtheit der Turksprachen gemeint.

3 Auch bei 10 und 11 hat es das türkische Wort, aber nicht als Suffix abtrennbar. 
in der Bedeutung zum Sanskritwort genauer zu passen als das mongolische Wort. Skt. -äha ist in der Weiterbildung qulaxu wiederzuerkennen (LC, $110 \mathrm{f}$.).

2. Skt. kokāha: türk. kök "blau" ist verbreitete Pferdefarbe in mehreren Dialekten ("fahl, gelblichgrau; silbergrau; eisengrau; Rotschimmel; grau”, alles von Pferden, LC, 79).

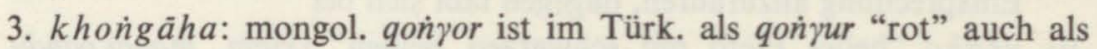
Pferdefarbe üblich (LC, 100). Für das Fehlen des $-r$ der altaischen Wörter im Skt. mit Mayrhofer das neuind. Substrat verantwortlich zu machen, scheint mir nicht angängig. Eher wird man an ein Suffix denken dafür scheinen die ewenkischen Entsprechungen konnomo neben konnorin, konnorui "schwarz", die N. Poppe in seiner Vergleichenden Grammatik der altaischen Sprachen (p. 99) gibt, zu sprechen. Die Bedeutung "schwarz" mag befremden, macht aber andererseits die Einbeziehung von

4. khu ṅgāha "Rappe, dunkles Pferd" diskutabel, das auch Mayrhofer nicht gern von khoñgāha trennen möchte. Vielleicht handelte es sich ursprünglich um die Bezeichnung eines komplexen Farberlebnisses, wie sie bei Reitervölkern häufig und dem Laien oft nur schwer durchschaubar sind.

5. serāha: für türk. sarï, sarï $\gamma$, dialektisch auch särï $\gamma$, seriq, säri, "blaß, bleich, blond, gelb” gibt LC, 65 keinen Beleg für Pferde, aber zahlreiche für Haare; dagegen bietet Radloff, Vergleichendes Wörterbuch der Türk-Dialecte, IV, 319 sar'ala at "Farbschecken" und $a k$ sari "ein Falbe", 300, sowie die Verwendung bei anderen haarigen Tieren (Gemse, Feuermarder, Zieselmaus). Zum Vokal der zweiten Silbe ist zu bemerken, daß das türk. $i$ wenigstens dem kurzen $a$ des Skt. sehr ähnlich ist, doch mag hier im Skt. der Anklang an die anderen -āhaWörter mitgewirkt haben.

6. haläha: türk. ala "bunt", im Anatol. auch hilä, ist als "gescheckt" für Pferde belegt (LC, $70 \mathrm{ff})$ und häufig in der Erweiterung alağa. Dieses $\check{g}$ ist nicht nur mit dem $-h$ - des Skt., sondern auch mit dem $q(\gamma, g)$ des Mongol. identisch. Näher als ein "Abfall” des $q$, den Mayrhofer hier annimmt, hätte im Neuindischen ein Stützvokal gelegen.

7. ukanāha hat auch im Türk. keine Entsprechung.

8. vollāha: dem von Mayrhofer nach Poucha angegebenen kalmück. borldã entspricht im Türk. būrul, pürul "mit gemischten Haaren, vom Pferd gesagt" (LC, 106f.). Wenn man von den Varianten mit anaptyktischem Ausfall des zweiten Vokals, tat. burli "mäusefarben", burlu 
"grau (Pferd)" ausgeht, kann das Sanskritwort als leicht verständliche Assimilation aus *borlāha gedeutet werden.

Für die folgenden Pferdewörter fehlen bei Mayrhofer noch Deutungen. Ich weiß bei

9. kiyāha "Fuchs" (lohita) auch aus dem Türkischen keine überzeugende Entsprechung anzuführen, dagegen läßt sich bei

10. uräha "helles Pferd mit schwarzen Beinen" (Hem. manäkpänḍu, krșnajangha) an türk. örüg "weiß, hell”, Nebenform zum yörü denken (LC, 49).

11. triyūha "kastanienbraunes Pferd (Hem. kapila)": deutlich <türk. toru $\chi$, tori $\chi$ "rotbraun (nur von Pferden)" (LC, 106). Im YasinBurushaski (alias Werchikwar) habe ich das Wort als turū $\gamma$ aufgezeichnet, wo es zunächst wohl aus dem Khowar entlehnt ist. Das darauf reimende

12. surūhaka "eselfarbiges Pferd (Hem. gardabhäbha)", von Mayrhofer zu Unrecht als "nicht hierhergehörig" in die Anmerkung (A 10) verwiesen, gehört wohl zu türk. sur "grau (von Pferden und anderen Tieren)", <*suru, das mittelbar noch in kirg. suručaq "Sperling" und tatar. sørø "grau" erhalten ist, vgl. LC, p. 111, bes. A 4. Die Herleitung aus pers. surx "rot" im $P W$, der sich Mayrhofer anschließt, ist wegen der Bedeutung viel weniger überzeugend.

Die Klärung lautlicher Einzelheiten der hier vorgeschlagenen Gleichungen und eine vielleicht mögliche Zuordnung zu einem bestimmten Turkdialekt muß ich dem Altaisten von Fach überlassen. Zur Möglichkeit einer Entlehnung aus dem Türkischen kann aber noch bemerkt werden, da $\beta$ direkter Kontakt zwischen Indern und Türken von alters her in Dardistan, dem nordwestlichen Teil des indoarischen Sprachgebiets, möglich war. Zwar ist das indoarische Gebiet dort von den Turksprachen immer noch durch einen etwa $250 \mathrm{~km}$ breiten Streifen mit iranischen Dialekten geographisch getrennt, doch zeigen die zahlreichen türkischen Entlehnungen neueren Datums in den Dard-Sprachen und dem nichtarischen Buruśaski, daß der Verkehr mit dem türkischen Norden zu allen Zeiten rege war. Aus dem Mongolischen hingegen, das ja auch von Dardistan viel weiter entfernt ist, scheinen keine neueren Entlehnungen in die genannten Sprachen gedrungen zu sein. 